\title{
MALDI-TOF mass spectrometry applied to identifying species of insect-pathogenic fungi from the Metarhizium anisopliae complex
}

\author{
Rogério Biaggioni Lopes, Marcos Faria, Daniela Aguiar Souza, Carlos Bloch \\ Jr., Luciano P. Silva \& Richard A. Humber
}

To cite this article: Rogério Biaggioni Lopes, Marcos Faria, Daniela Aguiar Souza, Carlos Bloch Jr., Luciano P. Silva \& Richard A. Humber (2014) MALDI-TOF mass spectrometry applied to identifying species of insect-pathogenic fungi from the Metarhizium anisopliae complex, Mycologia, 106:4, 865-878, DOI: 10.3852/13-401

To link to this article: https://doi.org/10.3852/13-401

\section{+ View supplementary material $๘$}

\section{Published online: 20 Jan 2017.}

Submit your article to this journal

$$
\text { Џll Article views: } 57
$$


Mycologia, 106(4), 2014, pp. 865-878. DOI: 10.3852/13-401

(C) 2014 by The Mycological Society of America, Lawrence, KS 66044-8897

\section{MALDI-TOF mass spectrometry applied to identifying species of insect-pathogenic fungi from the Metarhizium anisopliae complex}

\author{
Rogério Biaggioni Lopes \\ Marcos Faria \\ Daniela Aguiar Souza \\ Carlos Bloch Jr. \\ Luciano P. Silva \\ Embrapa Genetic Resources and Biotechnology, Brasilia \\ DF, CP 02372, 70770-917, Brazil \\ Richard A. Humber ${ }^{1}$ \\ USDA-ARS Biological Integrated Pest Management \\ Research, Robert W. Holley Center for Agriculture and \\ Health, 538 Tower Road, Ithaca, New York 14853
}

Abstract: Matrix-assisted laser desorption/ionization time-of-flight mass spectrometry (MALDI-TOF MS) has proven to be a powerful tool for taxonomic resolution of microorganisms. In this proof-of-concept study, we assessed the effectiveness of this technique to track the current gene sequence-based phylogenetic classification of species in the Metarhizium anisopliae complex. Initially the phylogenetic analysis of 51 strains by sequencing of the $5^{\prime}$ end of the $T E F-1 \alpha$ gene region revealed seven species within $M$. anisopliae sensu lato and two varieties outside this complex. Because initial studies on MS profiles from different cell types showed that mycelial fragments or conidia produced on nutrient-poor medium may yield too much background noise, all subsequent spectrometric analyses were performed with acidhydrolyzed conidia from 10-12 d old PDA cultures. The initial MALDI-TOF reference library included protein spectral profiles from nine taxonomically distinct, molecularly identified isolates sharing high genetic homology with the ex-type or ex-epitype isolates of these taxa in Metarhizium. A second reference library added one isolate each for $M$. anisopliae sensu stricto and $M$. robertsii. The second, larger reference library (including 11 taxa) allowed nearly perfect MALDI-TOF matching of DNA-based species identification for the 40 remaining isolates molecularly recognized as $M$. anisopliae sensu stricto $(\mathrm{n}=19)$, M. robertsii $(\mathrm{n}=6)$, M. majus $(\mathrm{n}=3), M$. lepidiotae $(\mathrm{n}=1)$, M. acridum $(\mathrm{n}=3)$, M. flavoviride var. pemphigi $(\mathrm{n}=1)$, plus seven unidentified strains (six of them phylogenetically close to $M$. anisopliae sensu stricto and one outside the Metarhizium

Submitted 24 Dec 2013; accepted for publication 5 Feb 2014.

${ }^{1}$ Corresponding author. E-mail: richard.humber@ars.usda.gov pingshaense-anisopliae-robertsii-brunneum clade). Due to the increasing frequency of phylogenetically (genomically) based taxonomic revisions of fungi, this approach is especially useful for culture collections, because once the protein profiles of Metarhizium isolates are obtained taxonomic updating of MALDI-TOF library data is easily accomplished by comparing stored profiles with those of newly proposed taxa.

Key words: Ascomycota, biocontrol, Clavicipitaceae, insect fungi, methodology, mycoinsecticides, phylogeny

\section{INTRODUCTION}

Classical taxonomy based on morphological characters is now widely understood to pose a series of challenges, including poor resolution and variability of traits, also requiring relatively lengthy identification processes (Santos et al. 2010). The taxonomic classification or identification of fungi has undergone major advances in the past decades, mainly following the adoption of molecular approaches (Hibbett et al. 2007). Phylogenetically based species complexes have been resolved and taxonomic revisions proposed for a number of insect fungal pathogens (Zare and Gams 2001, Zare et al. 2001, Luangsa-ard et al. 2004, Rehner and Buckley 2005, Sung et al. 2007, Bischoff et al. 2009, Rehner et al. 2011). Works focusing on multilocus phylogenetic analyses have clarified phylogenetic relationships within the hypocrealean genera Metarhizium (Bischoff et al. 2009) and Beauveria (Rehner et al. 2011) and yielded well justified proposals of new taxa within species complexes.

Microbial culture collections are valuable sources of authenticated germplasm for basic and applied research, for studies on biodiversity and systematics and also may serve as secure repositories for industrially important strains (Santos and Lima 2001, Smith 2003). Government culture collections, such as the USDA-ARS Collection of Entomopathogenic Fungal Cultures (ARSEF; Ithaca, New York) and the Invertebrate Fungal Collection at Embrapa Genetic Resources and Biotechnology (Brasilia DF, Brazil), hold large numbers of insect fungi from economically important species complexes, whose taxa show few or no clear morphological differences. Such collections are challenged to assure that their catalogs reflect current, well justified classifications 
that incorporate ongoing phylogenetically based taxonomic revisions. While almost all current molecularly based changes derive from gene sequence comparisons, alternative molecular profile technologies, such as matrix-assisted laser desorption/ionization time-of-flight mass spectrometry (MALDI-TOF MS), can allow low-cost, rapid and reliable identification for a wide range of microorganisms (Santos et al. 2010, Croxatto et al. 2012) including bacteria (Anhalt and Fenselau 1975, Carbonnelle et al. 2011), yeasts (Qian et al. 2008, Marklein et al. 2009, Bader et al. 2011, Pinto et al. 2011) and such taxonomically difficult genera of filamentous fungi as Fusarium (Seyfarth et al. 2008, Dong et al. 2009, Kemptner et al. 2009, Marinach-Patrice et al. 2009), Aspergillus (Li et al. 2000, Hettick et al. 2008, Rodrigues et al. 2011), Penicillium (Welham et al. 2000, Chen and Chen 2005) and Trichoderma (de Respinis et al. 2010, Samuels et al. 2010). However, this approach has not been applied to insect fungi until now.

The availability of practical and reliable identification techniques that also would allow rapid reclassification without relatively expensive and time consuming (PCR-based) re-analyses potentially could improve the quality of taxonomic information provided by fungal culture collections worldwide, including those concentrated on insect fungi. Therefore, the present proof-of-concept study seeks to test whether MALDI-TOF MS can be adjusted for the reliable tracking of gene sequence-based identification within the economically important and globally distributed Metarhizium anisopliae species complex.

\section{MATERIALS AND METHODS}

Source of fungi and their cultivation.-Fifty-one strains from the genus Metarhizium were used. Isolates with code CG (n = 39) were drawn from the Invertebrate Fungal Collection research team (Embrapa Genetic Resources and Biotechnology, Brasilia DF, Brazil); the other isolates were from the USDA-ARS Collection of Entomopathogenic Fungal Cultures (ARSEF 324, ARSEF 1914, ARSEF 1946, ARSEF 2024, ARSEF 2575, ARSEF 3391) and Instituto de Patologia Tropical e Saúde Pública (Universidade Federal de Goiás, Brazil) (IP 30, IP 60, IP 119, IP 123, IP 143, IP 146) (TABLE I). With the exception of eight isolates (all six ARSEF isolates plus CG374 and 645), all isolates had a Brazilian origin. These isolates were selected as being apparently representative of the Invertebrate Fungal Collection in terms of genetic diversity and included insect pathogens from orders Hemiptera, Coleoptera, Orthoptera, Lepidoptera and Hymenoptera or isolated from soil samples. Some strains were selected because they are active ingredients of commercial mycoinsecticides or because they currently are being used in some of our ongoing research projects (Lopes et al. 2013a, b). Isolate CG1123, whose genotype excludes this fungus from the so-called PARB clade (M. pingshaense-anisopliae-robertsii-brunneum) within the M. anisopliae species complex (Lopes et al. 2013b), was included as a further test of the robustness of MALDI-TOF profiles in comparison to sequence-based analyses.

Strains were inoculated on potato dextrose agar (PDA; Acumedia-Neogen Corp., Lansing, Michigan, and maintained at $25 \pm 0.5 \mathrm{C}$ and $12 \mathrm{~h}$ of photophase. Five-day-old culture plugs $(8 \mathrm{~mm}$ diam) were inoculated into $500 \mathrm{~mL}$ Erlenmeyer flasks containing $250 \mathrm{~mL}$ Sabouraud dextrose yeast broth (SDY broth; $1 \%$ glucose, $0.3 \%$ malt extract, $0.5 \%$ peptone, $0.3 \%$ yeast extract), followed by a $4 \mathrm{D}$ incubation at $25 \pm 0.5 \mathrm{C}$ in a rotary shaker at $250 \mathrm{rpm}$. Mycelial samples were harvested on filter paper by vacuum filtration, frozen at $-70 \mathrm{C}$, and then lyophilized and stored at $-20 \mathrm{C}$ until use. For MALDI-TOF studies, conidial samples $(4 \mathrm{mg})$ were gently collected from 10-12 d old cultures with a glass rod, avoiding fragments of mycelia and culture medium, and immediately transferred to $2 \mathrm{~mL}$ Eppendorf tubes containing $1 \mathrm{~mL} \mathrm{75 \%} \mathrm{ethanol.} \mathrm{The} \mathrm{suspension} \mathrm{was} \mathrm{vortexed} \mathrm{for} 30 \mathrm{~s}$ and centrifuged for $2 \mathrm{~min}$ at $16100 \times g$. The supernatant was discarded, and pellets were air-dried at room temperature for $15 \mathrm{~min}$ and maintained at $-12 \mathrm{C}$ until use.

Molecular identification of Metarhizium strains.-Molecular studies were performed to select strains sharing high homology with ex-type/ex-epitype isolates mentioned by Bischoff et al. (2009), so these strains could be further used for creation of main spectrum profiles (MSPs) in the MALDI-TOF MS studies. For each strain, approximately $25 \mathrm{mg}$ frozen hyphae were crushed in a mortar under liquid nitrogen and the total genomic DNA was extracted and processed further as described by Lopes et al. (2013a). Phylogenetic identification was performed by amplifying the $5^{\prime}$ end of $T E F-1 \alpha$ (translation elongation factor $1 \alpha$ ) gene fragment. Sequencing was performed by Macrogen Inc. (Seoul, South Korea) and edited with DNA baser (DNABaser Sequence Assembler 3, Heracle Biosoft, Pitesti, Romania). Consensus sequences were analyzed with MEGA 5.03 software (Tamura et al. 2011), and the final alignments were adjusted manually. Selected $T E F-1 \alpha$ sequences from GenBank for all strains originally preserved at the USDAARS Collection of Entomopathogenic Fungal Cultures and Instituto de Patologia Tropical e Saúde Pública were included in the analyses. In addition, other Metarhizium sequences from the same database were used as references in the phylogenetic tree (FIG. 1). Despite high consensus between GenBank and our own sequences for some cases in which both were available for creation of phylogenetic trees, GenBank sequences were given priority over our own sequence data.

Creation of the main spectrum profile (MSP).—Conidial pellets of each of 11 reference strains were resuspended in $50 \mu \mathrm{L}$ formic acid $(70 \%)$, vortexed $1 \mathrm{~min}$ and exposed to a 15-min cycle in a bath-type ultrasonic washer (Unique, model USC-1800, Indaiatuba, São Paulo, Brazil). Afterward, $50 \mu \mathrm{L}$ acetonitrile (100\%) were added to the mixture, vortexed $1 \mathrm{~min}$ and sonicated for additional $15 \mathrm{~min}$ before centrifugation $(16100 \times \mathrm{g}$ for $2 \mathrm{~min})$. The supernatant was diluted with deionized water $(1: 10 \mathrm{v} / \mathrm{v})$, and $1 \mu \mathrm{L}$ supernatant was directly spotted onto a MSP96 steel target 
plate (Bruker Daltonics GmbH, Bremen, Germany) and allowed to air-dry for $15 \mathrm{~min}$. A $1 \mu \mathrm{L}$ droplet of the $\alpha$-cyano4-hydroxycinnamic acid (CHCA) matrix solution (previously prepared by dissolving $10 \mathrm{mg}$ matrix powder [Bruker Daltonics $\mathrm{GmbH}$ ] in $100 \mu \mathrm{L}$ trifluoroacetic acid (TFA 3\%) + $400 \mu \mathrm{L}$ deionized water $+500 \mu \mathrm{L}$ acetonitrile) was applied over the dried sample and air-dried $15 \mathrm{~min}$. Analyses were performed on a MicroFlex LRF MALDI-TOF mass spectrometer (Bruker Daltonics $\mathrm{GmbH}$ ) with a nitrogen laser $(337 \mathrm{~nm})$ with $20-65 \%$ intensity and operating in spiral acquisition mode. A protein extract of Escherichia coli (Bruker Daltonics GmbH) was used for external calibration of this equipment. Each spectrum was obtained after an average of 240 laser shots (40 laser shots at six different spot positions) at $60 \mathrm{~Hz}$, and signals were automatically collected with the AutoXecute tool of the FlexControl acquisition software (3.3; Bruker Daltonics $\mathrm{GmbH}$ ) at a mass range of 2000-20 $000 \mathrm{~m} / \mathrm{z}$ under linear mode. Spectra were used in further analyses when the peaks showed a resolution higher than 200. Twenty-four independent samples (four conidial samples collected from six different cultures) were analyzed for each selected strain. Data were automatically exported to the Biotyper software (3.0; Bruker Daltonics $\mathrm{GmbH}$ ) and each consensus spectrum incorporated into the main spectrum profile (MSP) database. The consensus spectrum consists of a profile created from the peaks retrieved from at least 20 spectra collected under the same conditions from independent samples of any given isolate. The software performs all smoothing, normalization, baseline subtraction and peak-picking operations, thereby creating a list of the most significant peaks ( $\mathrm{m} / \mathrm{z}$ values) for each spectrum and their corresponding intensities. Peak frequency in all spectra for MSP creation was at least $25 \%$.

Effect of conidial quantity on MALDI-TOF mass spectral signals._Different conidial amounts (2.0, 4.0 or $20 \mathrm{mg}$ ) from 10-12 d old cultures of three Metarhizium species (M. robertsii CG520; M. pingshaense CG1091, M. anisopliae CG1125) were used in this preliminary experiment. Conidia from individual strains grown on PDA were carefully harvested and processed as explained above. Each sample was spotted onto a MSP96 steel target plate in triplicate prior to spectral acquisition.

Effects of mycelial fragments and growth media for conidial production.-Mycelial fragments from 3-day-old cultures, a mixture of mycelium and conidia from 6-day-old cultures (ca. 1:1), or only conidia from 10-day-old cultures of the same Metarhizium strains mentioned in the previous subsection, all grown on PDA, were subjected to MALDITOF MS analyses as previously described. M. anisopliae CG1125 also was grown on minimal medium (MM; $0.6 \%$ $\mathrm{NaNO}_{3}, 0.15 \% \quad \mathrm{KH}_{2} \mathrm{PO}_{4}, 0.05 \% \quad \mathrm{MgSO}_{4} \cdot 7 \mathrm{H}_{2} \mathrm{O}, 0.05 \% \mathrm{KCl}$, $0.0001 \% \mathrm{FeSO}_{4}, 0.0001 \% \mathrm{ZnSO}_{4}, 1 \%$ glucose, $1.5 \%$ agar) or Sabouraud dextrose agar amended with yeast extract (SDAY; $1 \%$ glucose, $0.3 \%$ malt extract, $0.5 \%$ peptone, $0.3 \%$ yeast extract, $1.5 \%$ agar) to assess the effect of different media on the mass spectral signals. Conidia from $10 \mathrm{~d}$ old cultures of M. anisopliae CG1125 were collected and processed as described for MALDI-TOF MS analyses. Germination percentages of fresh conidia harvested from the three solid media were assessed before acid digestion. Briefly, conidial suspensions $(20 \mu \mathrm{L})$ were pipetted onto PDA and Petri dishes were sealed with parafilm after water evaporation from suspension droplets and then incubated at $25 \pm 0.5 \mathrm{C}$ in darkness for $18 \mathrm{~h}$. Each treatment was repeated four times, and 300 conidia were scored in each replicate at $400 \times$ magnification. A conidium was considered to have germinated if the germ tube was at least as long as the length of an ungerminated conidium.

Validation of MALDI-TOF MS for identification of Metarhizium species.-All 51 Metarhizium strains grown on PDA were analyzed by MALDI-TOF mass spectrometry. Sample preparation was the same as described for pure conidia, and each strain was spotted in triplicates onto a MSP96 steel target plate. Data were processed with Biotyper 3 software, and all peaks in the mass range 3000-15000 m/z were considered. The collected spectra were processed with baseline subtraction (multipolygon) and Savitsky-Golay smoothing. The number of relevant peaks in mass spectra was limited to 100 , and only peaks with a signal/noise ratio $>3$ were used. The spectra were analyzed with the standard pattern-matching algorithm to match the number and intensity of peaks in the raw spectra with those in the reference libraries. Results of the pattern-matching process of the tested strains spectra against the MSP database were expressed as $\log$ (score) values in a $0-3$ scale, and those $<1.70$ (average for three independent samples) were rated by us as "unidentified". In addition, spectra of all 51 strains were submitted to cluster analysis (MSP dendrogram creation standard method 1.4) performed by Biotyper software. This analysis calculates the distance between values by a correlation function and creates a dendrogram from the calculated distance values by a statistical algorithm in this manufacturer's proprietary software.

\section{RESULTS}

Molecular identification of Metarhizium strains.Bayesian and maximum likelihood phylogenetic analyses produced similar topologies, with Metarhizium strains distributed in seven species within the $M$. anisopliae complex and one species outside this complex (TABLE I, FIG. 1). Species comprising the PARB ( $M$. pingshaense-anisopliae-robertsii-brunneum) clade proposed by Bischoff et al. (2009) were the most common: $M$. anisopliae sensu stricto $(\mathrm{n}=21), M$. robertsii $(\mathrm{n}=8), M$. pingshaense $(\mathrm{n}=1)$ and $M$. brunneum $(\mathrm{n}=1)$. M. anisopliae sensu stricto segregated into groups A and B, clearly clustering with ARSEF 6347 and the ex-type isolate, ARSEF 7487, respectively (FIG. 1). Our Group C (FIG. 1) comprised six strains (five comparatively similar and one more genetically divergent isolate) that are genetically close to $M$. anisopliae sensu stricto but that could not be confidently assigned to that species. Likewise two other groups of isolates identifiable genotypically as M. robertsii (groups D, E), clustered with strains 


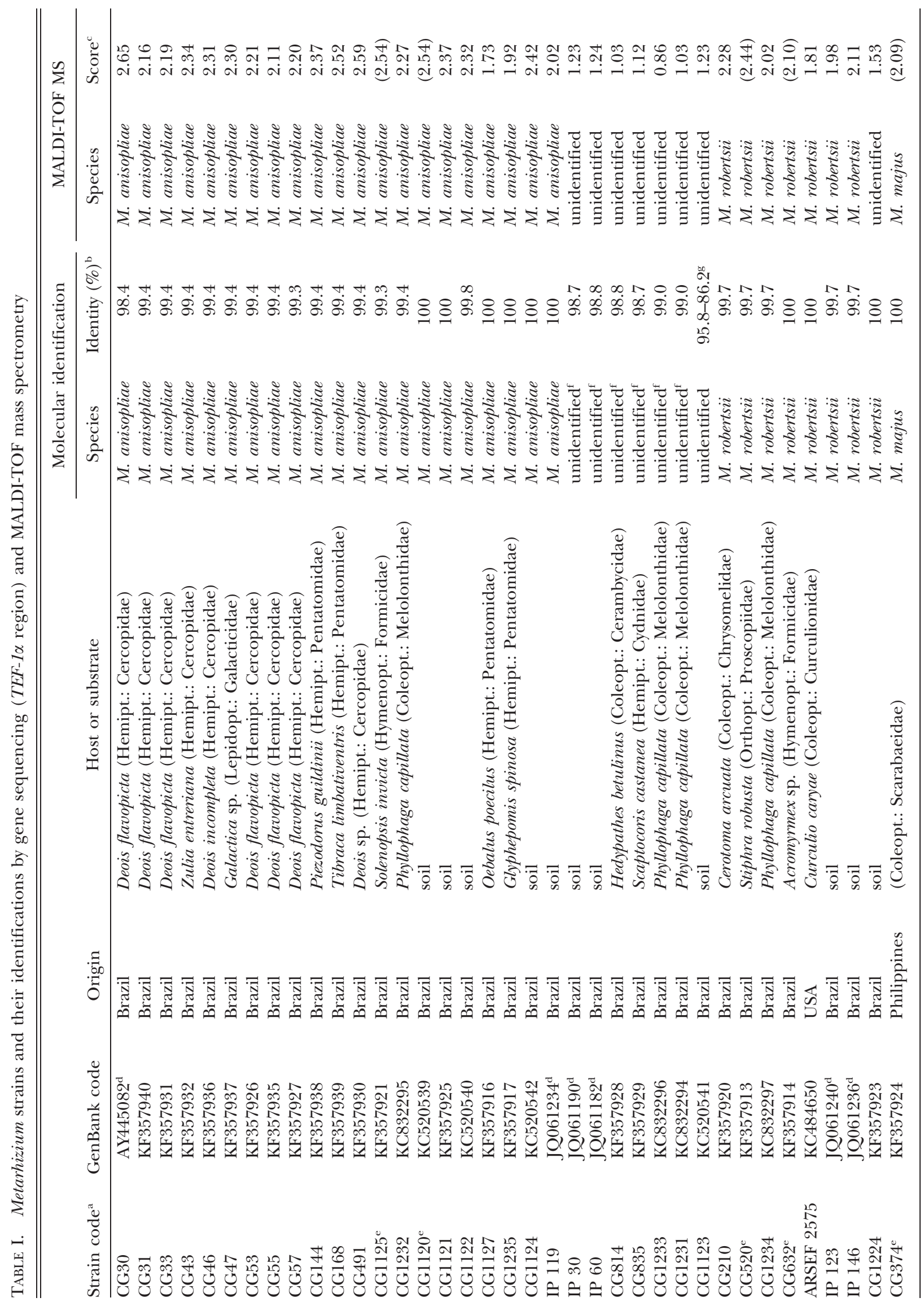




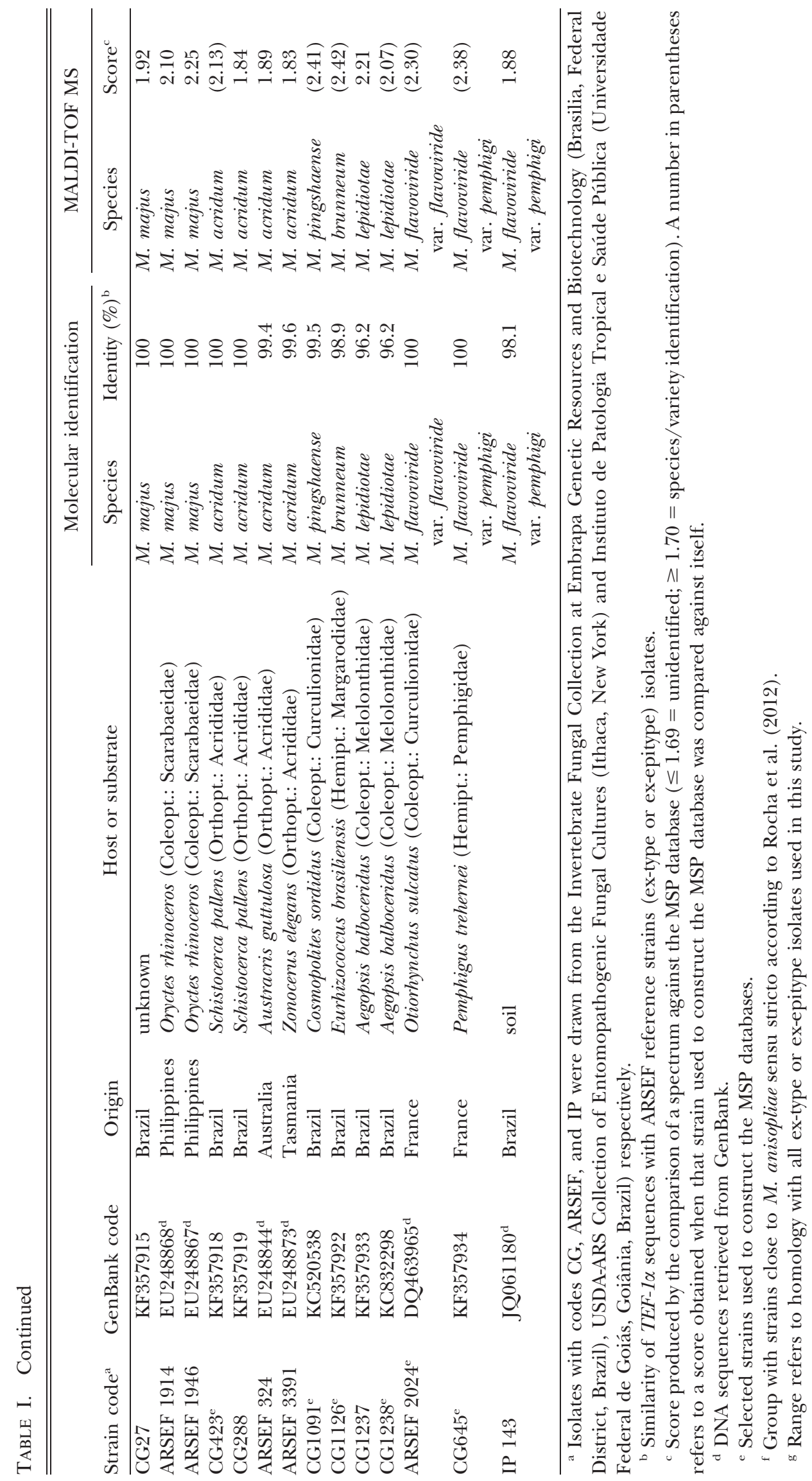




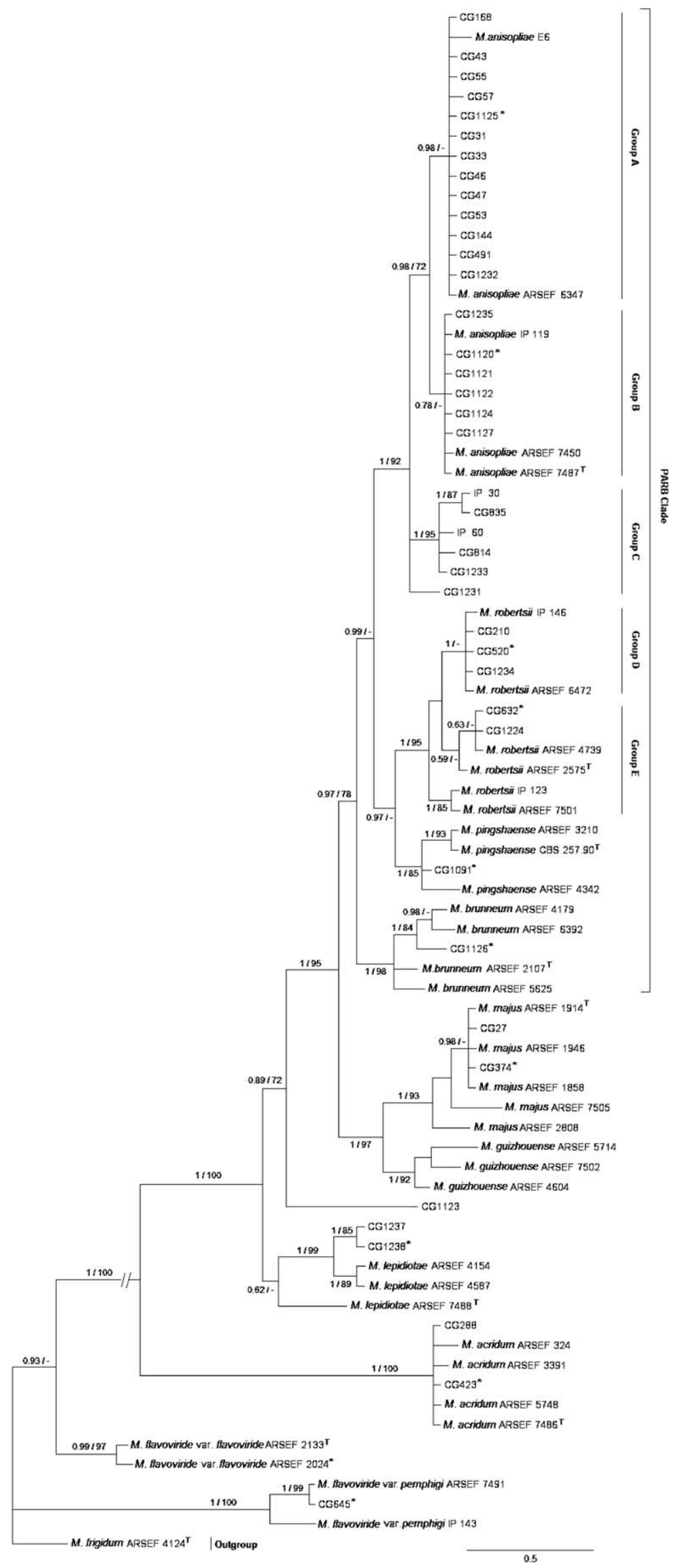




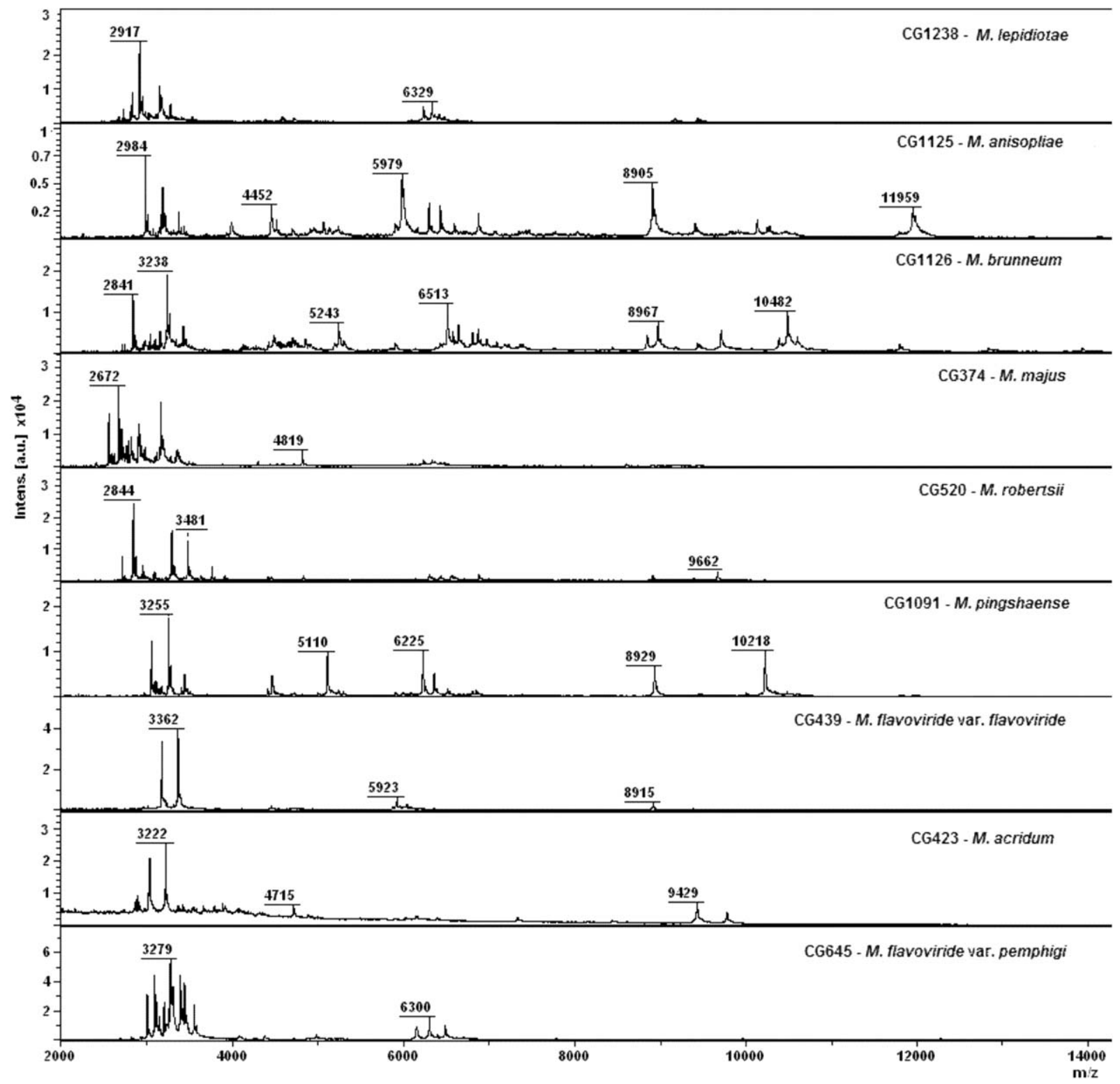

FIG. 2. Comparative differences among matrix-assisted laser desorption ionization-time-of-flight (MALDI-TOF) mass spectra for different Metarhizium species.

ARSEF 6472 and the ex-type isolate, ARSEF 2575, respectively. Outside the PARB clade, M. majus $(\mathrm{n}=$ 4), M. lepidiotae $(\mathrm{n}=2)$, and M. acridum $(\mathrm{n}=4)$ isolates clustered with strains ARSEF 1858, ARSEF 4154 and ARSEF 7486 respectively. In addition to these representatives of the $M$. anisopliae complex,
M. flavoviride var. flavoviride $(\mathrm{n}=1)$ and $M$. flavoviride var. pemphigi $(\mathrm{n}=2)$ also were identified, clustering with strains ARSEF 2133 and ARSEF 7491 respectively. Isolate CG1123, with a genotype from outside the PARB clade (Lopes et al. 2013b) and used here as a further control for MALDI-TOF profiles,

FIG. 1. Bayesian majority rule consensus phylogram of aligned $5^{\prime}$ end of TEF-1 $\alpha$ gene sequence data for Metarhizium strains. Support values were given as the Bayesian prior probability (first number) and percentage of bootstrap support derived from a MP analysis (second number). Isolates marked with a $\mathrm{T}$ are ex-type; those marked with an asterisk were used to construct the main spectrum profiles. 


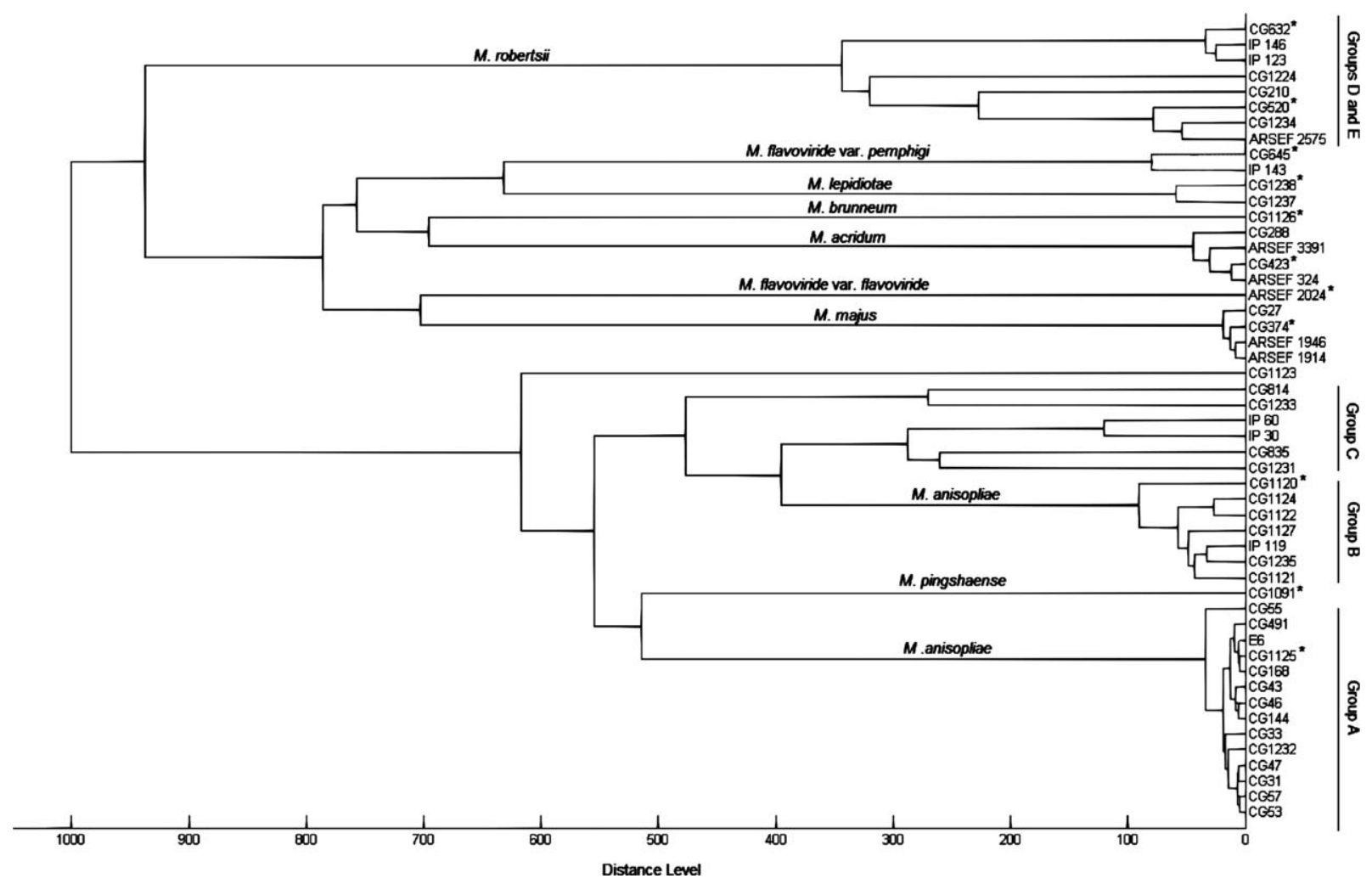

FIG. 3. Dendrogram showing cluster analysis of MALDI-TOF mass spectra. Isolates marked with an asterisk were used to construct the main spectrum profiles.

returned a protein profile that, as should be expected, did not match any known species in our study.

Creation of the main spectrum profile (MSP).-Based on the phylogenetic analysis, nine strains with high homology to corresponding ex-type or ex-epitype strains initially were selected for reference spectrum acquisition aiming creation of reference libraries. These isolates were M. anisopliae CG1125 (Group B; see FIG. 1 or TABLE I), M. robertsii CG520 (Group D), M. pingshaense CG1091, M. brunneum CG1126, M. majus CG374, M. lepidiotae CG1238, M. acridum CG423, M. flavoviride var. flavoviride ARSEF 2024, and M. flavoviride var. pemphigi CG645. The raw spectra of these nine species adopted in the first reference library are illustrated (FIG. 2). A second reference library also was built to include representation of all clearly identified taxa and groups by adding strains from groups A (M. anisopliae sensu stricto CG1120) and E (M. robertsii CG632).

Influences of conidial quantity, culture media and fungal cell types on MALDI-TOF MS mass spectral signals. - The quantity of conidia used in the hydrolysis process did not interfere with MALDI-
TOF signal quality for the recognition of $M$. robertsii CG520, M. pingshaense CG1091 or M. anisopliae CG1125 in that $\log$ (score) values were $\geq 2.10$ (SUPPLEMENTARY FIG. 1). On the other hand, mycelial samples produced low quality spectra and interfered with identification of all three strains, with score values in the $0.24-0.84$ range, values that were considerably below the cut-off limit of 1.70 (SUPPLEMENTARY FIG. 2). Although the use of mixed samples (mycelial fragments + conidia) was not problematic for $M$. robertsii CG520 or $M$. pingshaense CG1091, the $\log$ (score) value for the mixed sample was only 1.56 for $M$. anisopliae CG1125, whereas score values of this isolate obtained from only conidia were always $\geq 2.39$. Last, the initial viability of conidia harvested from cultures of M. anisopliae on SDAY, PDA and MM and used in the MALDI-TOF MS analyses were 99.5\%, $99.2 \%$ and $63.5 \%$ respectively. Spectra obtained from M. anisopliae CG1125 conidia produced on SDAY and PDA matched the reference library, with scores of 2.13 and 2.20 respectively. Growth on MM produced a slightly different profile and a much lower identification score of 1.39 (SUPPLEMENTARY FIG. 3).

Validation of MALDI-TOF MS for identification of Metarhizium species.-When the original 9 strain 
reference library was used, no strains from $M$. anisopliae sensu stricto and $M$. robertsii groups $\mathrm{A}$ and E (groups not containing isolates with high genetic homology to the respective ex-type or exepitype strains) could be reliably identified because their average $\log$ (scores) were considerably below the cut-off value of 1.70. However, when we adopted the more comprehensive library-the original nine isolates plus one additional reference strain from both Groups A and E-accurate MALDI-TOF identifications were obtained for all 19 non-reference isolates of $M$. anisopliae sensu stricto and for five of the six nonreference $M$. robertsii strains tested.

In addition to the 11 strains in the expanded reference library, MALDI-TOF profiles were generated for 40 additional test strains of Metarhizium. Robust matches with the reference strains (with score values $\geq 1.70$ ) were obtained for 39 out of 40 test strains. $M$. robertsii CG1224 was the only strain whose genomically determined identity was not unambiguously confirmed by mass spectrometry despite repeated analyses of this isolate. Despite its relatively low score against the $M$. robertsii reference isolate, the MS profile of CG1224 is more similar to the profiles of $M$. robertsii than to any other taxon tested here. As might be expected, the seven isolates whose genetic identifications were not available all yielded inconclusively low $\log$ (scores) $0.86-1.24$. Because only single strains of $M$. pingshaense, M. brunneum and $M$. flavoviride var. flavoviride were available for the MALDI-TOF study, these species were still incorporated into the reference libraries but the effectiveness of this technique for diagnosing these species has not been adequately tested; the addition of further strains will certainly improve the reliability of the MALDI-TOF approach for identifying these taxa. Further, a group of genetically similar Brazilian strains (IP 30, IP 60, CG814, CG835, CG1231, CG1233) that are also genetically similar to but not verifiably identical with M. anisopliae sensu stricto proved to have protein spectra forming their own distinct cluster, labeled here as Group C (FIG. 3). The taxonomic status of these isolates clearly deserves further evaluation with multigenic phylogenetic studies. The lack of MSbased correspondence of strain CG1123 with any other species used here similarly underscores the need for further gene-based characterization of this strain.

The dendrograms of protein-based MALDI-TOF MS identification profiles closely matched those of the gene-based phylograms from Bischoff et al. (2009). This mass spectrometric approach successfully recovered groupings of taxa that are essentially identical to those from genomic analyses. Nonetheless, the relative distances and placements of taxo- nomic groups among these MALDI-TOF profiles differed from those in their gene sequence-based analyses (e.g. FiG. 1, and Bischoff et al. 2009).

\section{DISCUSSION}

Fungal identification by MALDI-TOF mass spectrometry is based on comparisons of protein spectrum profiles of unidentified strains against the reference profiles of known taxa. While it is inherently a molecular technique, MALDI-TOF differs significantly from the direct comparisons of gene sequences used for phylogenetically based systematics. Despite the universal dependence on sequence-based systematics as a methodological approach for revising fungal systematics and discovering phylogenetic relationships, it is useful to remember that MALDI-TOF profiles show differences among large numbers of unidentified proteins reflecting a much larger proportion of a total organismal genome than is now routinely used with multigenic sequence-based studies. These two dramatically different approaches should be accepted as useful in their own contexts. In this study, we created main spectrum profiles for reference strains (those with high genetic homology to ex-type and ex-epitype isolates) that allowed accurate MALDI-TOF identifications (agreeing with identifications based on the $T E F-1 \alpha$ gene fragment) of 39 out of 40 non-reference strains from a number of species within the Metarhizium anisopliae complex. We do not believe and do not wish to imply that MSbased identification of fungi can or should replace their gene-based identifications that have become the de facto required standard for almost all taxonomic studies.

The robustness of the MALDI-TOF MS approach for Metarhizium species identification is highly influenced by both sample preparation procedures (as shown here) and by diverse sorts of contaminants affecting mass spectrometers (Keller et al. 2008). We adjusted a protein extraction method from aerial conidia, but the presence of hyphae (mycelial fragments) in the test preparation can influence the spectral signal and, therefore, its matching against the standard reference profiles. These results confirmed the importance of standardizing growth conditions and other methodologies before MALDI-TOF analyses and also underscore previous studies that proteins are differentially expressed by conidia and mycelium, as was shown for the protein-expression profiles of $M$. acridum mycelium and conidia in which only $35 \%$ of the proteins were common to both cell types (Barros et al. 2010). Studies of filamentous fungi by MALDITOF MS based on vegetative cells usually yielded satisfactory identifications for species of Trichoderma 
(de Respinis et al. 2010); these authors concluded that MALDI-TOF MS analyses of $2 \mathrm{~d}$ old mycelial samples were useful for species delimitation in this genus. Similarly Chowdappa et al. (2013) showed significant MALDI-TOF capacity to identify Alternaria species by analyzing intact $5 \mathrm{~d}$ old mycelium. However, we observed both higher quality and better reproducibility for protein spectra using only conidia of Metarhizium. Nonetheless, conidia produced on a low-nutrient medium (MM) showed both different protein profiles and poorer germination than those from high-nutrient media (PDA or SDAY). Although conidia formed on nutritionally marginal substrates may not be so completely developed as those on nutritionally richer substrates, the more important observation for Metarhizium species is that the most informative proteins for compiling MALDI-TOF profiles were obtained from conidia only, rather than from mycelium or mycelial/conidial mixtures.

This study highlighted the importance of using the broadest possible selection of representative strains to create reference libraries. Libraries based only on extype or ex-epitypes isolates (or, as was our case, on isolates showing high genetic homology to these taxonomic standard isolates) did not support unequivocal identifications of a number of unknown strains. However, when we added several more isolates of $M$. anisopliae sensu stricto and $M$. robertsii isolates to our MS-based molecular studies, we were able to distinguish clearly two subgroups within each of these two species. By adding representatives of these two groups not previously considered (CG1125 and CG520 of M. anisopliae sensu stricto Group A and $M$. robertsii Group E respectively), non-reference strains from these taxa used in this work could be robustly identified by spectrometric analyses. In general, a high degree of gene sequence similarities between unknown isolates and their corresponding ex-type/ex-epitypes was correlated with MALDI-TOF MS scores $\geq 2.00$ (a default value commonly used in similar studies). Exceptions found here were the three $M$. acridum strains, with genetic homology in the $99.4-100 \%$ range but that had MALDI-TOF MS scores in the 1.83-1.89 range, and with five other seemingly anomalous isolates. Both CG1127 and CG1235 (M. anisopliae Group A; see FIG. 1) showed $100 \%$ genetic homology but MALDI-TOF scores with their correct group of only 1.73 and 1.92 respectively. IP 123 (M. robertsii Group E) showed 99.7\% genetic homology but a MALDI-TOF score of 1.98; CG27 ( $M$. majus) showed $100 \%$ genetic homology with its extype but a score of 1.92; and IP 143 (M. flavoviride var. pemphigi) showed $98.1 \%$ genetic homology but a score of 1.88. Although MALDI-TOF MS scores for $M$. acridum were slightly below the default value for highly confident MS-based identifications, these strains clearly grouped with the ex-type isolate, ARSEF 7486 , in the phylogenetic analysis, all were obtained from acridid hosts and their morphological features (e.g. their conidial dimensions and pale colony color) also were consistent with their traditional gene-based identification as M. acridum. Therefore, the lower cutoff value adopted in our study (1.70) was supported by morphological traits. This integration of morphological, genetic sequence and protein profiling approaches has been referred to as an advantageous strategy for the reliable identification of fungi (Wicht et al. 2012).

A notable exception to these criteria for identification was isolate CG1224 (M. robertsii Group E), which showed $100 \%$ genetic homology to its corresponding ex-type isolate but a MALDI-TOF score of only 1.53. CG1224 was the only example we encountered of a strain that could not be spectrometrically matched with its genotypically identified reference. Nonetheless, its MS profile was closer to the M. robertsii strains used to construct the MSP database than to any other tested taxon. Since our expectation in this study was to achieve matching of identifications between MS and the current genetically based classification system, we could have chosen a lower cut-off value (e.g. $\leq 1.53$ ), but the adoption of a more stringent limit gave us the confidence that all other MS identifications were correct and also provided stronger clues about which isolates need further sequenced-based taxonomic evaluation.

Among the seven strains that were tested without genomically confirmed identifications, six of them, including isolates IP 30 and IP 60 (Rocha et al. 2012) and CG1231 and CG1233 (Lopes et al. 2013a), belong to a sister group related to M. anisopliae (s.l.), clustered together and are designated here as Group C. According to these studies, additional molecular characterization will be required to determine whether these isolates might represent a new taxon. Indeed, our Group C isolates appear to be similar to another group of Brazilian isolates that is already being more intensively studied with a multigenic sequence approach (S. Rehner pers comm). No isolate from this group was added to the main reference library because those libraries were specifically intended to include only fungi identified definitively by genomic characters. We think that Group C probably represents an undescribed cryptic species of Metarhizium but would not include any isolate from this group in the reference library until more complete gene-based studies can confirm this probable status. If any Group $\mathrm{C}$ isolate were placed into the main reference library, all remaining isolates belonging to its group would be retrieved with significantly high score values as identifiable with that reference isolate. 
TABLE II. Comparative estimates of time and reagent costs between single-gene phylogeny and mass spectrometric analyses (MALDI-TOF) for each group of 100 isolates within the Metarhizium anisopliae complex

\begin{tabular}{|c|c|c|}
\hline Processing step & DNA sequencing & MALDI-TOF \\
\hline \multicolumn{3}{|l|}{ Initial growth and processing } \\
\hline Cultivation of fungus on solid medium & $10-12 \mathrm{~d}$ & $10-12 \mathrm{~d}$ \\
\hline Extraction of proteins from conidia & - & $2 \mathrm{~d}$ \\
\hline Protein processing & - & $<6 \mathrm{~h}$ \\
\hline Cultivation of fungi in liquid medium & $3 \mathrm{~d}$ & $-^{\mathrm{a}}$ \\
\hline \multicolumn{3}{|l|}{ First analysis } \\
\hline Mycelial processing & $2-3 \mathrm{~d}$ & $-^{\mathrm{a}}$ \\
\hline DNA extraction & $2-3 \mathrm{~d}$ & $-^{\mathrm{a}}$ \\
\hline PCR amplification & $1-2 \mathrm{~h}$ & ${ }^{\mathrm{a}}$ \\
\hline Sequencing/purification or MALDI-TOF ${ }^{\mathrm{b}}$ & $3-4 \mathrm{~h}$ & $2-3 \mathrm{~h}$ \\
\hline Data analyses & $1-3 \mathrm{~d}$ & $<1 \mathrm{~h}$ \\
\hline Estimated total time for processing & $18-24 \mathrm{~d}$ & $12-14 \mathrm{~d}$ \\
\hline Estimated cost for reagents ${ }^{b}$ & US\$ 515.00 & US\$ 2.00 \\
\hline $\begin{array}{l}\text { Re-analysis to accommodate new phylogenetic } \\
\text { reclassification }\end{array}$ & same as above & $\begin{array}{l}\text { only for reference isolates } \\
\text { from new taxa }\end{array}$ \\
\hline
\end{tabular}

${ }^{\text {a }}$ For the MALDI-TOF approach, gene sequencing is usually unnecessary, unless in the rare occasions in which one or more reference isolates used to compile the Main System Profile library have not been sequenced before (as was the case in the present study).

${ }^{\mathrm{b}}$ Contrary to the sequencing approach, only reference isolates from new taxon/taxa have to be subjected to MALDI-TOF when a new phylogenetic classification is proposed, followed by data analyses with all isolates (including those protein profiles previously stored).

${ }^{\mathrm{c}}$ Estimated cost in US\$ for analyzing 100 isolates at the Invertebrate Fungal Collection (Brasilia, Brazil) considering steps following conidial/mycelial processing. Estimates for reagents were based on concentrations adopted in this publication. Additional costs, such as labor (1-2 people) and equipment (acquisition/maintenance), were not included. Based on our experience, commercial services of sequencing/purification would cost ca. US $\$ 1200.00$ per 100 isolates.

Another strain, CG1123, isolated from soil in a banana plantation, was known to be within the $M$. anisopliae complex but not within the PARB clade (Lopes et al. 2013b). This fungus was not unambiguously identifiable by either gene sequencing or spectrometric analyses and needs further multiple gene-based evaluation as a possible new taxon. Further resolution of the genus Metarhizium outside the PARB clade (Kepler et al. 2014) also is required to unravel both the phylogeny and specific identifications of this fungus; once the corresponding main mass spectrometric protein profiles are generated for Metarhizium species outside the $M$. anisopliae species complex, MALDI-TOF should become an even more valuable tool to flag individual isolates that do not fit well within the current taxonomy of this genus and are candidates for more complete characterization and possible description as new cryptic taxa.

While MALDI-TOF MS and gene sequencing resulted in highly similar groupings within Metarhizium, spectrometry did not perfectly reflect gene sequence-based phylogeny (Bischoff et al. 2009); a similar result also was observed for some Trichoderma clades by de Respinis et al. (2010) and for Alternaria species by Brun et al. (2013). The apparently close degree of phylogenetically determined relatedness of the sister groups M. anisopliae (s.str.) and M. robertsii (Bischoff et al. 2009) was not mirrored by MALDITOF analyses; these taxa are well distinguished by their MALDI-TOF profiles but were placed relatively distant from each other on the MS-based dendrogram (FIG. 3). Indeed, M. anisopliae groups A and B were separated by a $M$. pingshaense strain (CG1091) whereas $M$. robertsii groups $\mathrm{D}$ and $\mathrm{E}$ clustered together but at the opposite extreme of the dendrogram and, therefore, in conflict with the grouping of the PARB clade seen in gene-based phylogenetic trees (FIG. 1; also see Bischoff et al. 2009). It is likely that the use of more specifically targeted and larger numbers of molecular markers in addition to those now commonly used for fungal phylogenetics will better define variability within the $M$. anisopliae complex, as was recently shown by Kepler and Rehner (2013). Note, however, that MALDI-TOF confirms the assignments of isolates to each of these groups and that the strength of this spectrometric technique is in providing basic specific identification with a high degree of confidence. The degree of phylogenetically determined relatedness between these groups (whether or not they represent discrete taxa) is an 
appropriate question to ask of sequence-based studies but is emphatically not a relevant issue for the use of MALDI-TOF mass spectrometry. The utility of MALDI-TOF is limited to taxa for which good reference libraries of verifiably identified and generally closely related (congeneric) isolates are compiled; it cannot serve adequately to identify random, wholly unidentified isolates. There is no overall reference database of MALDI-TOF profiles comparable to GenBank or other genomic databases against which BLAST queries with a single gene sequence may allow more or less reasonably meaningful identifications.

The relatively low operational cost and rapidity of the spectrometric approach in comparison to gene sequencing deserves careful evaluation in that PCR and sequencing (including commercial services) are more or less globally ubiquitous and available at everdecreasing costs. We must recognize first, however, that the capital expense for either DNA sequencers or the mass spectrometric equipment for MALDI-TOF analyses is prohibitively high for most individual laboratories. These methodological approaches may be most practical if individual scientists are able to send their material to properly equipped and maintained central facilities; this practice is already common for obtaining DNA sequences. Compelling evidence (TABLE II) reveals that, apart from the high costs of the hardware for either sequence-based or MS studies, MALDI-TOF approaches indeed are both rapid and inexpensive in a point-by-point comparison of the time and costs for on-demand processing of isolates by $\mathrm{PCR} /$ gene sequencing and MALDI-TOF techniques. Keeping in mind that the overall goals may be quite different in choosing to use one over the other of these two approaches, there can be little question that MALDI-TOF can provide routine, day-today insights (with the trade-off of a slightly lower confidence) about identifications both faster and less expensively than the use of PCR and sequencing technologies. In our opinion, a balanced view is that MALDI-TOF would be economically feasible and advisable for institutions (e.g. many large culture collections) maintaining many hundreds or thousands of isolates from single genera and that also have reasonable access to appropriately equipped massspectrometric facilities. In these cases, investments related to spectrometer acquisition could be (at least partially) compensated over time by providing authentication services to research and industrial customers and also should allow the rapid improvements for the quality of identifications given to many isolates in those collections that were originally and are still identified only by their morphological characteristics.

Based on our own experiences in curating government-owned culture collections, the validation of
MALDI-TOF MS for preliminary species identifications with a high degree of confidence for entomopathogenic (and, eventually, other) fungi is a major accomplishment. In spectrometric analyses, strains would need to be analyzed only once and, whenever necessary, taxonomic updating of an entire collection after phylogenetic reclassifications is easily and rapidly accomplished by comparing stored profiles with those of newly proposed taxa. In contrast, the addition of one or more new gene sequences in any genomically based taxonomic revision nominally would require the longer, more expensive, and more complex task of sequencing of all affected isolates for the new gene(s) to verify or to adjust their identifications. The satisfactory degree of taxonomic accuracy reported here is vital when recommending this comparatively rapid approach as an alternative to gene sequencing for preliminary screening of fungal biodiversity, ecology and population biology that, by their very nature, often tend to involve dauntingly large numbers of isolates.

\section{ACKNOWLEDGMENTS}

We thank Drs Stephen Rehner and Ryan Kepler (USDAARS, Beltsville, Maryland) for their kind cooperation in providing supplemental information and sequence data.

\section{LITERATURE CITED}

Anhalt JP, Fenselau C. 1975. Identification of bacteria using mass spectrometry. Anal Chem 47:443,219-225, doi:10.1021/ac60352a007

Bader O, Weig M, Taverne-Ghadwal L, Lugert R, Grob U, Kuhns M. 2011. Improved clinical laboratory identification of human pathogenic yeasts by matrix-assisted laser desorption ionization time-of-flight mass spectrometry. Clin Microbiol Infect 17:1359-1365.

Barros BHR, Silva SH, Marques ER, Rosa JC, Yatsuda AP, Roberts DW, Braga GUL. 2010. A proteomic approach to identifying proteins differentially expressed in conidia and mycelium of the entomopathogenic fungus Metarhizium acridum. Fungal Biol 114:572579, doi:10.1016/j.funbio.2010.04.007

Bischoff JF, Rehner SA, Humber RA. 2009. A multilocus phylogeny of the Metarhizium anisopliae lineage. Mycologia 101:508-528, doi:10.3852/07-202

Brun S, Madrid H, van den Ende BG, Andersen B, Marinach-Patrice C, Mazier D, de Hoog GS. 2013. Multilocus phylogeny and MALDI-TOF analysis of the plantpathogenic species Alternaria dauci and relatives. Fungal Biol 117:32-40, doi:10.1016/j.funbio.2012. 11.003

Carbonnelle E, Mesquita C, Bille E, Day N, Dauphin B, Beretti J, Ferroni A, Gutmann L, Nassif X. 2011. MALDI-TOF mass spectrometry tools for bacterial identification in clinical microbiology laboratory. Clin 
Biochem 44:104-109, doi:10.1016/j.clinbiochem. 2010.06.017

Chen HY, Chen YC. 2005. Characterization of intact Penicillium spores by matrix-assisted laser desorption/ ionization mass spectrometry. Rapid Commun Mass Spectrom 19:3564-3568, doi:10.1002/rcm.2229

Chowdappa P, Lakshmi M, Jyothi M, Madhura S. 2013. Matrix assisted laser desorption ionization-time of flight (MALDI-TOF) mass spectrometry for identification of plant pathogenic Alternaria species. Phytoparasitica 41:169-179, doi:10.1007/s12600-012-0276-7

Croxatto A, Prod'hom G, Greub G. 2012. Applications of MALDI-TOF mass spectrometry in clinical diagnostic microbiology. FEMS Microbiol Rev 36:380-407, doi:10.1111/j.1574-6976.2011.00298.x

de Respinis S, Vogel G, Benagli C, Tonolla M, Petrini O, Samuels GJ. 2010. MALDI-TOF MS of Trichoderma: a model system for the identification of microfungi. Mycol Prog 9:79-100, doi:10.1007/s11557-009-0621-5

Dong H, Kemptner J, Marchetti-Deschmann M, Kubicek CP, Allmaier G. 2009. Development of a MALDI two-layer volume sample preparation technique for analysis of colored conidia spores of Fusarium by MALDI linear TOF mass spectrometry. Anal Bioanal Chem 395:13731383, doi:10.1007/s00216-009-3067-3

Driver F, Milner RJ, Trueman JWH. 2000. A taxonomic revision of Metarhizium based on a phylogenetic analysis of rDNA sequence data. Mycol Res 104:134150, doi:10.1017/S0953756299001756

Hettick JM, Green BJ, Buskirk AD, Kashon ML, Slaven JE, Janotka E, Blachere FM, Schmechel D, Beezhold DH. 2008. Discrimination of Aspergillus isolates at the species and strain level by matrix-assisted laser desorption/ionization time-of-flight mass spectrometry fingerprinting. Anal Biochem 380:276-281, doi:10.1016/ j.ab.2008.05.051

Hibbett DS, Binder M, Bischoff JF, Blackwell M, Cannon PF, Eriksson O, Huhndorf S, James T, Kirk PM, Lücking R, Lumbsch T, Lutzoni F, Matheny PB, McLaughlin DJ, Powell MJ, Redhead S, Schoch CL, Spatafora JW, Stalpers JA, Vilgalys R, Aime MC, Aptroot A, Bauer R, Begerow D, Benny GL, Castlebury LA, Crous PW, Dai Y-C, Gams W, Geiser DM, Griffith GW, Gueidan C, Hawksworth DL, Hestmark G, Hosaka K, Humber RA, Hyde K, Köljalg U, Kurtzman CP, Larsson K-H, Lichtwardt R, Longcore J, Miadlikowska J, Miller A, Moncalvo J-M, Mozley-Standridge S, Oberwinkler F, Parmasto R, Reeb V, Rogers JD, Roux C, Ryvarden L, Sampaio JP, Schuessler A, Sugiyama J, Thorn RG, Tibell L, Untereiner WA, Walker C, Wang Z, Weir A, Weiss M, White M, Winka K, Yao Y-J, Zhang N. 2007. A higherlevel phylogenetic classification of the Fungi. Mycol Res 111:509-547, doi:10.1016/j.mycres.2007.03.004

Keller BO, Sui J, Young AB, Whittal RM. 2008. Interferences and contaminants encountered in modern mass spectrometry. Anal Chem Acta 627:71-81, doi:10. 1016/j.aca.2008.04.043

Kemptner J, Marchetti-Deschmann M, Mach R, Druzhinina IS, Kubicek CP, Allmaier G. 2009. Evaluation of matrixassisted laser desorption/ionization (MALDI) prepara- tion techniques for surface characterization of intact Fusarium spores by MALDI linear time-of-flight mass spectrometry. Rapid Commun Mass Spectrom 23:877884, doi:10.1002/rcm.3949

Kepler R, Rehner S. 2013. Genome-assisted development of nuclear intergenic sequence markers for entomopathogenic fungi of the Metarhizium anisopliae species complex. Mol Ecol Resour 13:210-217, doi:10.1111/ 1755-0998.12058

Li TY, Liu BH, Chen YC. 2000. Characterization of Aspergillus spores by matrix assisted laser desorption/ ionization time-of-flight mass spectrometry. Rapid Commun Mass Spectrom 14:2393-2400, doi:10.1002/ 1097-0231 (20001230) 14:24<2393::AID-RCM178>3.0. $\mathrm{CO} ; 2-9$

Lopes RB, Souza DA, Oliveira CM, Faria M. 2013a. Genetic diversity and pathogenicity of Metarhizium spp. associated with the white grub Phyllophaga capillata (Blanchard) (Coleoptera: Melolonthidae) in a soybean field. Neotr Entomol 42:436-438, doi:10.1007/s13744-0130146-0

—- Mesquita ALM, Tigano MS, Souza DA, Martins I, Faria M. 2013b. Diversity of indigenous Beauveria and Metarhizium spp. in a commercial banana field and their virulence toward Cosmopolites sordidus (Coleoptera: Curculionidae). Fungal Ecol 6:356-364, doi:10.1016/j.funeco.2013.06.007

Luangsa-Ard JJ, Hywel-Jones NL, Samson RA. 2004. The polyphyletic nature of Paecilomyces sensu lato based on 18S generated rDNA phylogeny. Mycol Res 109:581589, doi:10.1017/S0953756205002741

Marinach-Patrice C, Lethuillier A, Marly A, Brossas JY, Gené J, Symoens F, Datry A, Guarro J, Mazier D, Hennequin C. 2009. Use of mass spectrometry to identify clinical Fusarium isolates. Clin Microbiol Infec 15:634-642, doi:10.1111/j.1469-0691.2009.02758.x

Marklein G, Josten M, Klanke U, Muller E, Horré R, Maier T, Wenzel T, Kostrzewa M, Bierbaum G, Hoerauf A, Sahl HG. 2009. Matrix-assisted laser desorption ionization-time of flight mass spectrometry for fast and reliable identification of clinical yeast isolates. J Clin Microbiol 47:2912-2917, doi:10.1128/JCM.00389-09

Pinto A, Halliday C, Zahra M, van Hal S, Olma T, Maszewska K, Iredell JR, Meyer W, Chen SCA. 2011. Matrix-assisted laser desorption ionization-time of flight mass spectrometry identification of yeasts is contingent on robust reference spectra. PLoS ONE 6:e25712, doi:10.1371/ journal.pone.0025712

Qian J, Cutler JE, Cole RB, Cai Y. 2008. MALDI-TOF mass signatures for differentiation of yeast species, strain grouping and monitoring of morphogenesis markers. Anal Bioanal Chem 392:439-449, doi:10.1007/s00216-008-2288-1

Rehner SA, Buckley E. 2005. A Beauveria phylogeny inferred from nuclear ITS and EF1- $\alpha$ sequences: evidence for cryptic diversification and links to Cordyceps teleomorphs. Mycologia 97:84-98, doi:10.3852/mycologia.97.1.84

- Minnis A, Sung G, Luangsa-Ard JJ, Devotto L, Humber RA. 2011. Phylogeny and systematics of the anamorphic entomopathogenic genus Beauveria. Mycologia 103:1055-1073, doi:10.3852/10-302 
Rocha LFN, Inglis PW, Humber RA, Kipnis A, Luz C. 2012. Occurrence of Metarhizium spp. in central Brazilian soils. J Basic Microbiol 53:251-259, doi:10.1002/ jobm.201100482

Rodrigues P, Santos C, Venâncio A, Lima N. 2011. Species identification of Aspergillus section Flavi isolates from Portuguese almonds using phenotypic, including MALDI-TOF ICMS, and molecular approaches. J Appl Microbiol 111:877-892, doi:10.1111/j.1365-2672.2011. 05116.x

Samuels GJ, Ismaiel A, Bon M, de Respinis S, Petrini O. 2010. Trichoderma asperellum sensu lato consists of two cryptic species. Mycologia 102:944-966, doi:10.3852/ 09-243

Santos C, Paterson RRM, Venâncio A, Lima N. 2010. Filamentous fungal characterizations by matrix-assisted laser desorption/ionization time-of-flight mass spectrometry. J Appl Microbiol 108:375-385, doi:10.1111/ j.1365-2672.2009.04448.x

Santos IM, Lima N. 2001. Criteria followed in the establishment of a filamentous fungi culture collection, Micoteca da Universidade do Minho (MUM). World J Microbiol Biot 17:215-220, doi:10.1023/A:1016655631991

Seyfarth F, Ziemer M, Sayer HG, Burmester A, Erhard M, Welker M, Schliemann S, Straube E, Hipler U. 2008. The use of ITS DNA sequence analysis and MALDITOF mass spectrometry in diagnosing an infection with Fusarium proliferatum. Exp Dermatol 17:965-971, doi:10.1111/j.1600-0625.2008.00726.x
Smith D. 2003. Culture collections over the world. Int Microbiol 6:95-100, doi:10.1007/s10123-003-0114-3

Sung GH, Hywel-Jones NL, Sung JM, Luangsa-Ard JJ, Shreshtha B, Spatafora JW. 2007. Phylogenetic classification of Cordyceps and the clavicipitaceous fungi. Stud Mycol 57:5-59, doi:10.3114/sim.2007.57.01

Tamura K, Peterson D, Peterson N, Stecher G, Nei M, Kumar S. 2011. MEGA 5: molecular evolutionary genetics analysis using maximum likelihood, evolutionary distance and maximum parsimony methods. Mol Biol Evol 28:2731-2739, doi:10.1093/molbev/msr121

Welham KJ, Domin MA, Johnson K, Jones L, Ashton DS. 2000. Characterization of fungal spores by laser desorption/ionization time-of-flight mass spectrometry. Rapid Commun Mass Spectrom 14:307-310, doi:10.1002/ (SICI) 1097-0231 (20000315) 14:5<307::AID-RCM823> 3.0.CO;2-3

Wicht B, Orlando P, Mauro J. 2012. Molecular, proteomic and morphological characterization of the ascomycete Guignardia bidwellii, agent of grape black rot: a polyphasic approach to fungal identification. Mycologia 104:1036-1045, doi:10.3852/11-242

Zare R, Gams W. 2001. A revision of Verticillium section Prostrata IV. The genera Lecanicillium and Simplicillium. Nova Hedwigia 73:1-50.

- - Evans HC. 2001. A revision of Verticillium section Prostrata V. The genus Pochonia, with notes on Rotiferophthora. Nova Hedwigia 73:51-86. 